\title{
Abkürzungen und Vorbemerkungen
}

$\mathrm{A}=$ Codex Paris. Graec. 451 (914 geschrieben), s. S. XIII bis XVIII.

$B=$ Codex Paris. Graec. 465 (13. Jahrh.), s. S. XIX-XXIII.

$\mathrm{D}=$ Codex Paris. Graec. 467 (16. Jahrh.), s. S. XLIII-XLVII; nur subsidiarisch neben $\mathrm{N}$ verwendet, $\mathrm{s}$. S. XLVII.

$\mathrm{G}=$ Codex Laurent. Plut. VI 9 (1344 geschrieben), s. S. XXXVII bis XLI; nur subsidiarisch neben $\mathrm{O}$ verwendet, $\mathrm{s}$. S. XIII.

$\mathrm{H}=$ Codex Marc. 343 (11. Jahrh.); s. S. XVIIIf.; nur subsidiarisch neben A verwendet, s. S. XIX.

I = Codex Marc. 341 (15. Jahrh.), s. S. XXIII -XXXII.

$\mathrm{N}=$ Codex Neapol. II AA 16 (15. Jahrh.), s. S. XLIIf.

$\mathrm{O}=$ Codex Bononiens. 3643 (Endedes 13. Jahrh.), s. S. XXXIII bis XXXVII; OG bedeutet: eine in 0 unsichere Lesart wird durch $\mathrm{G}$ bestätigt.

$\mathrm{V}=$ Codex Batopedianus 180 (Anfang des 14. Jahrh.), s. S. XLVII-XLIX.

Unselbständige Hss.: S. XXVII, XXXIIf., XLIX.

$\mathrm{LXX}=$ Septuaginta

$\mathrm{NT}=$ Novum Testamentum.

Eine in Klammern neben der Sigle einer Hs. stehende Angabe bezieht sich nur auf diese Hs.

$\mathrm{A}^{*}$ (oder eine andere Sigle) $*$ die betreffende Hs. vor der Korrektur.

$\alpha$ (oder ein anderer Buchstabe) ausgebess(ert): die betreffende Hs. hat eine aus einer andern Lesart umgeänderte Lesart, z. B.

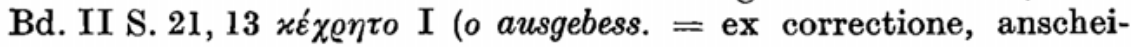
nend aus al).

nachgebess(ert): eine undeutlich gewordene Lesart ist wieder hergestellt worden.

verbess (ert): aus einer falschen ist die richtige Lesart hergestellt

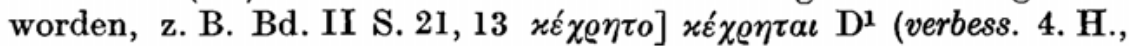
d.h. aus $x \varepsilon ́ \chi \varrho \eta \tau \alpha \iota$ ist das richtige $\chi \varepsilon ́ \chi \varrho \eta \tau o ~ g e m a c h t$ worden). 


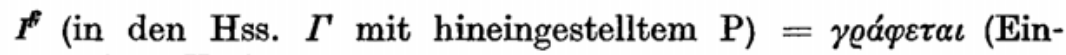
führung einer Variante).

$$
\begin{aligned}
& \alpha \text { (oder ein anderer Buchstabe) }=\alpha \text { unsicher. } \\
& \text {. = ein fehlender Buchstabe. } \\
& x=\text { Rasur eines Buchstabens. } \\
& +=\text { fügt (fügen) hinzu, hinzugefügt. } \\
& <=\text { läßt (lassen) aus. } \\
& \sim=\text { stellt (stellen) um. } \\
& \langle>=\text { einzufügen (fehlt in allen Hss.). } \\
& {[]=\mathrm{zu} \text { tilgen (steht in allen Hss.). }} \\
& \dagger=\text { Textverderbnis. } \\
& 1=\mathrm{im} \text { Text: Seitenschluß bei Vigerus. Im textkritischen }
\end{aligned}
$$

a. R. = am Rand.

i. T. = im Text.

nml. = nämlich.

Dind. $=$ Dindorf.

Gaisf. = Gaisford.

Giff. $=$ Gifford.

Hkl. = Heikel (s. S. XIII).

Schw. = Ed. Schwartz.

Steph. $=$ Robertus Stephanus.

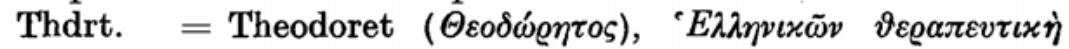
$\pi \alpha \vartheta \eta \mu \alpha ́ \tau \omega \nu$ (Graecarum affectionum curatio, rec. I. Raeder, Lipsiae 1904). S. s. L.

TU $=$ Texte und Untersuchungen zur Geschichte der altchristlichen Literatur.

Vig. = Vigerus; auf seine Ausgabe beziehen sich die auf den rechten Rändern stehenden Zahlen der durch (a), b, c, d abgeteilten Seiten, die Seitenenden seiner Ausgabe sind durch | bezeichnet. 